\title{
Whistler mode auroral hiss emissions observed near Jupiter's moon Io
}

\author{
L. Xin, ${ }^{1}$ D. A. Gurnett, ${ }^{1}$ and M. G. Kivelson ${ }^{2}$ \\ Received 7 September 2005; revised 8 December 2005; accepted 16 December 2005; published 21 April 2006.
}

[1] On 16 October 2001, the Galileo spacecraft made a close flyby of Jupiter's moon Io. During the flyby the plasma wave instrument detected an electric field emission with spectral characteristics very similar to a type of whistler-mode noise called "auroral hiss" that is commonly observed in Earth's auroral region. This paper gives a detailed analysis of the "auroral hiss" observed near Io. The frequency-time spectrum of the emission has a sharp high-frequency cutoff near the electron cyclotron frequency and a V-shaped lowfrequency cutoff. On a frequency-time spectrogram these cutoffs give the emission a characteristic funnel shape that is very similar to the spectrum of terrestrial auroral hiss. Strong magnetic field perturbations occurred near the vertex of the funnel indicating the presence of a field-aligned current. To explain the origin of the emission, a brief review is given of whistler-mode wave propagation and the unipolar inductor model of Io's interaction with the magnetosphere of Jupiter. Assuming propagation near the whistlermode resonance cone, ray-tracing analyses show that the radiation originates from a source very close to the surface of Io. The source is located in the same region where fieldaligned currents are believed to originate in the ionosphere of Io. Since terrestrial auroral hiss is known to be produced by beams of low-energy auroral electrons, these observations suggest that the auroral hiss at Io is generated by an electron beam that is part of the field-aligned current system induced by the interaction of Io with the rapidly rotating magnetosphere of Jupiter.

Citation: Xin, L., D. A. Gurnett, and M. G. Kivelson (2006), Whistler mode auroral hiss emissions observed near Jupiter's moon Io, J. Geophys. Res., 111, A04212, doi:10.1029/2005JA011411.

\section{Introduction}

[2] The objective of this paper is to study an unusual plasma wave emission observed by the Galileo spacecraft during a close flyby of Jupiter's moon Io that occurred on 16 October 2001. A frequency-time spectrogram showing the electric field intensities detected by the Galileo plasma wave instrument during this flyby is shown in Figure 1. The emission of interest is labeled "auroral hiss" and occurs from about 0116:30 to 0121:30 universal time (UT) and extends over a frequency range from about 2 to $40 \mathrm{kHz}$. The term "auroral hiss" arises from the close similarity to a whistler-mode emission frequently observed over Earth's auroral regions called auroral hiss. Terrestrial auroral hiss is known to be produced by field-aligned beams of low-energy (100 eV to several keV) auroral electrons [Gurnett, 1966; Gurnett and Frank, 1972]. Using ray tracing, we show that the auroral hiss originates very close to Io in the same region as the field-aligned current induced by the interaction of Io with the corotating magnetosphere of Jupiter.

\footnotetext{
${ }^{1}$ Department of Physics and Astronomy, University of Iowa, Iowa City, Iowa, USA.

${ }^{2}$ Institute of Geophysics and Planetary Physics, University of California, Los Angeles, Los Angeles, California, USA.

Copyright 2006 by the American Geophysical Union. 0148-0227/06/2005JA011411\$09.00
}

\section{Previous Studies of Auroral Hiss}

[3] The first reported observations of the high-latitude radio phenomena known as auroral hiss were from ground-based very-low-frequency (VLF) radio measurements [Martin et al., 1960]. More detailed studies of these emissions were subsequently carried out using a VLF radio receiver on the low-altitude polar-orbiting Injun 3 satellite [Gurnett, 1964; Gurnett, 1966]. These studies showed that auroral hiss was observed on almost every pass over the auroral zone at magnetic latitudes ranging from about 65 to 80 degrees and that the emissions were closely correlated with the aurora and with the low-energy $(\mathrm{keV})$ precipitating electrons responsible for the aurora. The satellite observations also showed that auroral hiss often has a characteristic Vshaped low-frequency cutoff that gives the emission a funnel shape on a frequency-time spectrogram. The Vshaped low-frequency cutoff is now known to be produced by whistler-mode propagation near the resonance cone [Mosier and Gurnett, 1969]. Later studies with the high-altitude polar-orbiting DE-1 spacecraft [Gurnett et al., 1983] showed that both upward and downward propagating auroral hiss can occur and that the upward propagating auroral hiss was produced by an upward moving beam of low-energy electrons with energies as low as $100 \mathrm{eV}$. Since these electron beams are the primary current carriers in the magnetic field-aligned currents that couple the aurora to the distant magneto- 


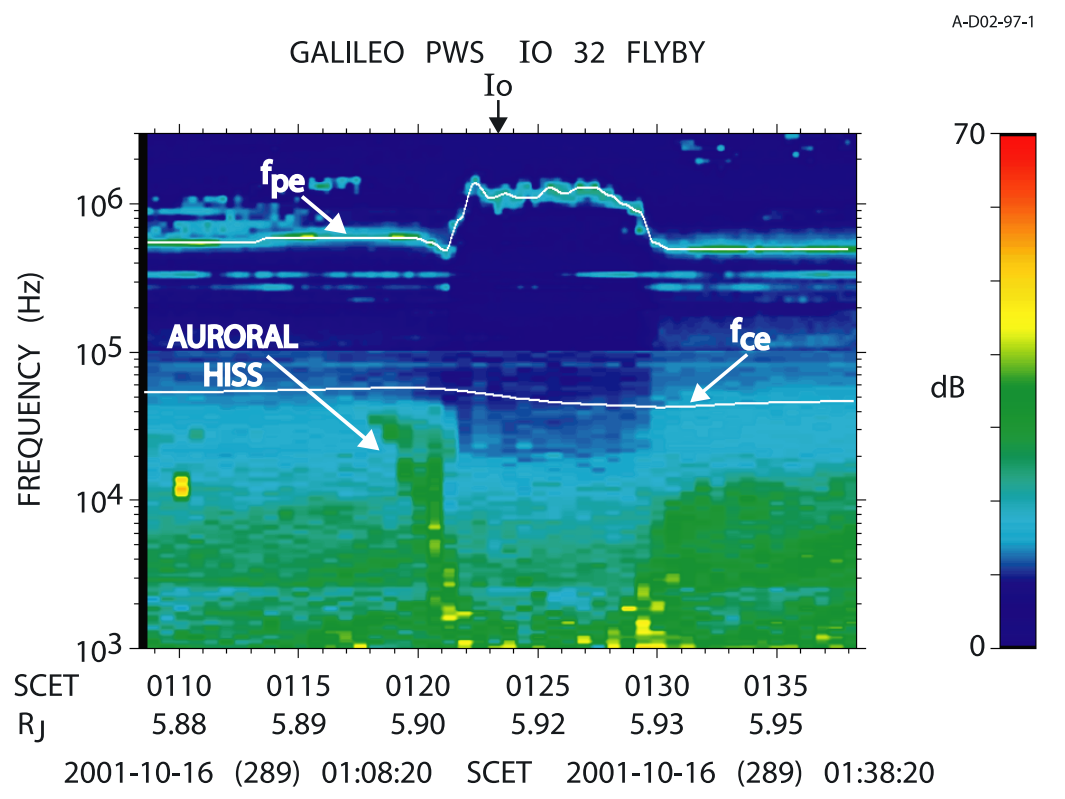

Figure 1. A frequency-time spectrogram of auroral hiss observed by the Galileo spacecraft near the south pole of Jupiter's moon Io. The intensities are color coded with red representing the strongest emission while the blue color represents the weakest emission. The dynamic range from dark blue to bright red is $70 \mathrm{~dB}$.

sphere, these observations showed that auroral hiss is closely associated with the large-scale field-aligned current system that drives the aurora.

[4] Auroral hiss has also been observed in the magnetosphere of Jupiter [Gurnett and Kurth, 1979; Farrell et al., 1993]. The Jovian auroral hiss has a characteristic V-shaped low-frequency cutoff that is similar to the auroral hiss observed over Earth's auroral regions. However, the Jovian auroral hiss was observed at very high altitudes, near the inner edge of the Io plasma torus. From an analogy with Earth-based observations, Gurnett and Kurth [1979] suggested that the emissions were generated by a field-aligned electron beam with energies from about $10 \mathrm{eV}$ to $1 \mathrm{keV}$ and fluxes in the range $10^{8}$ to $10^{10}$ electrons $\mathrm{cm}^{-2}$. Assuming whistler-mode propagation near the resonance cone, the Vshaped low-frequency cutoff of the Jovian auroral hiss was analyzed by both Gurnett and Kurth [1979] and Morgan et al. [1994] to determine the source location of the electron beam. They concluded that the electron beam originated near the inner edge of the hot plasma torus at $\mathrm{L}=5.59$ at a distance of about 0.58 Jovian radii $\left(\mathrm{R}_{\mathrm{J}}\right)$ from the centrifugal equator. They also suggested that the electron beam was probably part of a large-scale field-aligned current system induced by the interaction of the Io plasma torus with the corotating magnetic field of Jupiter.

[5] Various authors have attempted to explain how an electron beam can generate auroral hiss. Many of the early theories were based on an incoherent Cerenkov mechanism [Ellis, 1957; Jorgensen, 1968; James, 1973]. Cerenkov radiation is produced whenever the index of refraction in the medium is such that a charged particle can move faster than the phase velocity of the wave [Jackson, 1962]. This condition is easily satisfied for the whistler mode, since the index of refraction becomes very large along the cone of directions known as the resonance cone. Despite the obvious merits of this mechanism, Taylor and Shawhan [1974] showed that the observed intensities of auroral hiss are much too large to be explained by an incoherent mechanism and concluded that the emissions must be generated by a coherent plasma instability. The principles involved in such beam-driven instabilities are now well known and have been applied to the generation of auroral hiss by various authors, including for example Maggs [1976]. Some very interesting tests of the coherent beam-generation mechanism have been provided by space-borne electron beam experiments [Cartwright and Kellogg, 1974; Monson et al., 1976; Gurnett et al., 1986; Farrell et al., 1988]. One such experiment, an artificial electron beam with an energy of $1 \mathrm{keV}$ and a current of $50 \mathrm{~mA}$, was ejected from the space shuttle and the resulting plasma wave emissions were detected by a spacecraft called the Plasma Diagnostics Package (PDP) at distances of up to $250 \mathrm{~m}$ from the space shuttle. A frequency-time spectrogram showing the plasma wave electric field intensities observed as the PDP flew through the ejected electron beam is shown in Figure 2. An obvious funnel-shaped emission can be seen centered on the beam. Ray tracing computations showed that the V-shaped low-frequency cutoff agreed very well with the expected propagation of whistler-mode waves near the resonance cone. The measured wave powers were also found to be nearly $10^{7}$ greater than those expected from incoherent Cerenkov radiation, verifying that the radiation must be generated by a coherent plasma instability. A nonlinear computer plasma simulation performed by Farrell et al. [1989] later showed that a coherent plasma instability driven by the Landau reso- 


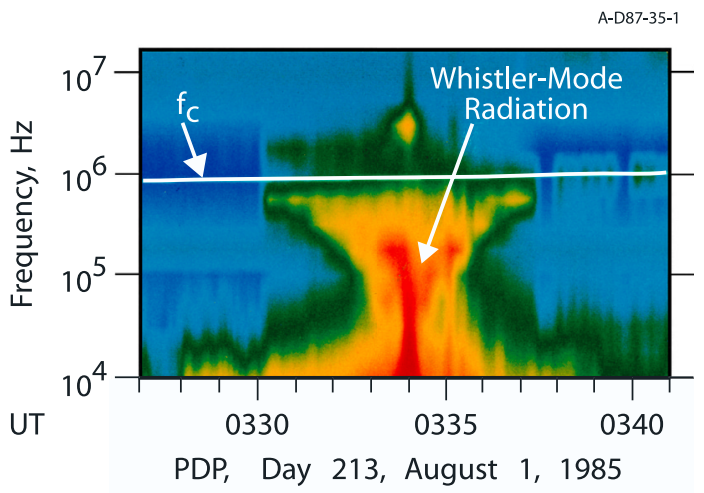

Figure 2. A frequency-versus-time spectrogram from the PDF plasma wave instrument. The funnel-shaped structure that extends from the electron cyclotron frequency to about $30 \mathrm{kHz}$ is whistler-mode radiation from the man-made electron beam (reprinted from Farrell et al. [1988]).

nance at $v_{\|}=\omega / k_{\|}$could easily account for the observed whistler-mode radiation intensities.

\section{Observations}

[6] The spacecraft trajectory for the 16 October 2001, flyby of Io is shown in Figure 3. This flyby occurred on orbit 32 and was the sixth close flyby of Io. The trajectory is plotted in an Io-centered coordinate system with the $+\mathrm{z}$ axis aligned parallel to Jupiter's rotational axis and the $+x$ axis aligned parallel to the nominal corotational plasma flow induced by Jupiter's rotation. The $+y$ axis completes the usual right-handed coordinate system. The spacecraft passed under the south pole of Io at 0123:20 UT with a closest approach radial distance of about $2000 \mathrm{~km}$.

[7] The spectrum in Figure 1 shows that the auroral hiss has an asymmetrical funnel-shaped low-frequency cutoff that decreases monotonically from about $40 \mathrm{kHz}$ at 0116:00 UT to about $1 \mathrm{kHz}$ at 0121:00UT. The white line marked $f_{c e}$ near the upper cutoff of the auroral hiss at about 43 to $60 \mathrm{kHz}$ is the electron cyclotron frequency. The electron cyclotron frequency was computed from onboard magnetic field measurements [Kivelson et al., 1996] using the equation $f_{c e}=28 \mathrm{~B} \mathrm{~Hz}$, where $B$ is the magnetic field strength in $\mathrm{nT}$. The corresponding proton cyclotron frequency is about 25 to $30 \mathrm{~Hz}$. The electron plasma frequency can be determined from the frequency of upper hybrid emission observed during this pass and is shown by the white line marked $f_{p e}$ in Figure 1. The electron plasma frequency is about $600 \mathrm{kHz}$ during the period when the auroral hiss was observed. From these observations the following inequalities exist among the proton cyclotron frequency $f_{c i}$, the emission frequency $f$, the electron cyclotron frequency $f_{c e}$, and the electron plasma frequency $f_{p e}$,

$$
f_{c i} \ll f \ll f_{c e}<f_{p e} .
$$

For these parameters the only possible mode of propagation in the frequency range of interest is the whistler mode [e.g., Gurnett and Bhattacharjee, 2005]. As will be shown shortly, these inequalities allow us to greatly simplify the cold plasma dispersion relation, which will be used later to perform ray-path calculations.

[8] Figure 4 shows plots of the $\mathrm{x}, \mathrm{y}$, and $\mathrm{z}$ components of the magnetic field as obtained from the onboard magnetometer for the same time range shown in Figure 1. From these plots, it can be seen that all three components of the magnetic field are smooth and slowly varying except in the interval between about 0115:00 and 0132:00 UT, which corresponds to the time range when the spacecraft was in the vicinity of Io (comparing with Figure 3). In this interval large perturbations are clearly observed in the $B_{x}$ and $B_{y}$ plots with amplitudes of about $\Delta B_{x} \approx 600 \mathrm{nT}$ (relative to a background of field of about $-300 \mathrm{nT}$ ) and $\Delta B_{y} \approx 300 \mathrm{nT}$ (again relative to a background of about $-300 \mathrm{nT}$ ). Obvious abrupt step-like changes in the magnetic field occurred at about 0120:30 and 0129:30 UT. According to Ampere's law $\nabla \times \mathbf{B}=\mu_{0} \mathbf{J}$, these changes indicate that the spacecraft crossed two intense magnetic field-aligned current sheets, one directed in approximately the $+\mathrm{z}$ direction near the inbound crossing of the Io flux tube and the other directed in the $-\mathrm{z}$ direction at about 0120:30 UT near the outbound crossing of the Io flux tube. In the region between the two current sheets, the z-component of magnetic field increased (decreased in magnitude) gradually with some small fluctuations. After the second current sheet crossing at 0129:30 UT, the $\mathrm{B}_{\mathrm{z}}$ field drops down to an equilibrium value of about $-1650 \mathrm{nT}$, which is slightly larger than the field $(-1900 \mathrm{nT})$ that was present during the approach to Io. These fieldaligned currents are almost certainly caused by the unipolar inductor interaction of Io with the rotating magnetosphere of Jupiter, as first proposed by Goldreich and Lynden-Bell [1969] or the closely related Alfvén wing interaction [Neubauer ,1980; Southwood et al., 1980]. Magnetic perturbations generated by the field-aligned currents were detected previously by Voyager 1 magnetic field measurements as the spacecraft crossed the Io flux tube at about 11 Io radii $\left(\mathrm{R}_{\mathrm{Io}}\right)$ south of Io [Ness et al., 1979]. Comparing

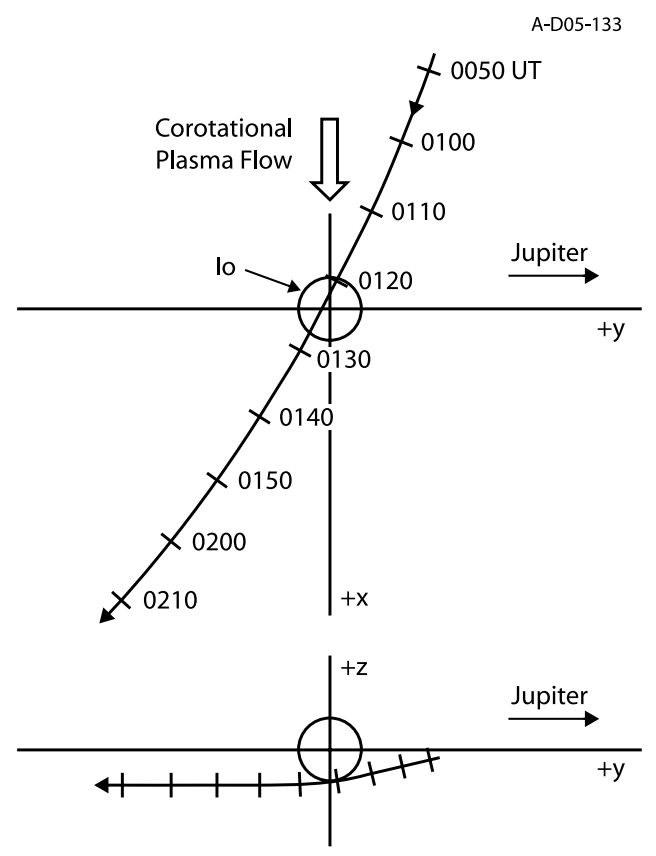

Figure 3. The trajectory of Galileo during the Io-32 flyby. 


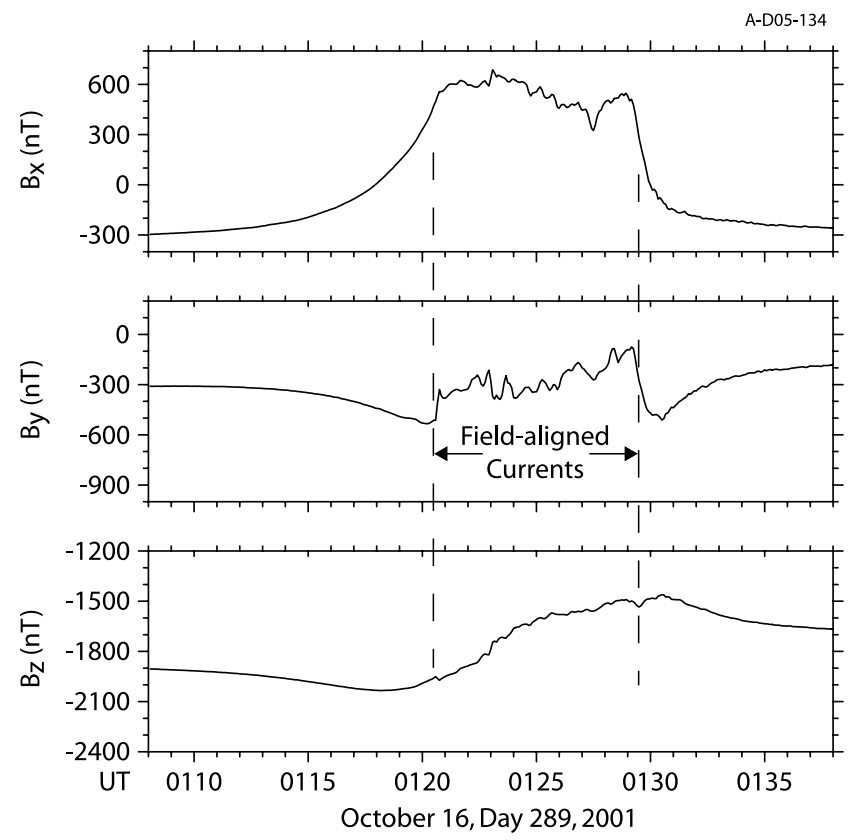

Figure 4. Magnetic field components measured by the Galileo Magnetometer on 16 October 2001 plotted versus spacecraft event time in UT.

the electric field spectrum in Figure 1 with the magnetic field variations in Figure 4, it is obvious that the vertex of the auroral hiss funnel occurs almost exactly at the same time as the inbound current sheet crossing, i.e., at 0120:30 UT. This close correspondence strongly suggests that the auroral hiss is associated with the inbound current sheet crossing. It is also noteworthy that there is no detectable auroral hiss associated with the outbound current sheet crossing, which is in the opposite direction.

\section{Theory of Auroral Hiss Propagation}

[9] The plasma temperature in the Io plasma torus is known to be very low, only about $10 \mathrm{eV}$ [Bagenal, 1994]. Since at this low temperature the phase velocity of the whistler mode is much greater than the electron thermal velocity, cold plasma theory is expected to provide a very good model for analyzing the propagation of whistler-mode waves in the vicinity of Io. Also, since the frequency of the auroral hiss is well above all of the ion cyclotron frequencies, the ion terms in the cold plasma dispersion relation can be ignored. For the plasma parameters that exist in the vicinity of Io, $f^{2} \ll f_{p}^{2}$ and $f_{c}^{2} \ll f_{p}^{2}$, it is easy to show [e.g., Helliwell, 1965; Gurnett and Bhattacharjee, 2005] that the index of refraction of the whistler mode is given to a good approximation by the equation

$$
n^{2}=\frac{f_{p}^{2}}{f\left(f_{c} \cos \theta-f\right)} .
$$

Because of ion effects, this equation is only valid for frequencies well above the lower hybrid frequency, $f_{L H}$, which in the vicinity of Io is a few hundred Hz. Polar plots of the index of refraction, $n$, as a function of the wave normal angle, $\theta$ are shown in Figure 5 for three different frequencies, $f_{1}, f_{2}$, and $f_{3}$. If these plots are rotated around the magnetic field they generate a surface called the index of refraction surface [Stix, 1992]. As can be seen in Figure 5, the index of refraction goes to infinity at an angle, $\theta_{\text {Res. }}$. This angle is called the resonance cone angle and defines a cone of directions around the magnetic field called the resonance cone. It is easy to show from equation (2) that the resonance cone angle is given by

$$
\cos \theta_{\text {Res }}=f / f_{c} .
$$

Since the group velocity, which is the direction of energy propagation, is always perpendicular to the index of refraction surface [Stix, 1992], it is easy to see that the limiting angle, $\psi$, between the group velocity direction and the magnetic field direction, is given by $\psi=\pi / 2-\theta_{\text {Res }}$. Using equation (3), the corresponding group velocity direction, which is also the ray path direction, is given by

$$
\sin \psi=f / f_{c} .
$$

[10] Consider now a point that is radiating whistler mode waves along the resonance cone at a ray path angle $\psi$ relative to the magnetic field $B_{0}$ as shown in the upper panel of Figure 6. It is obvious from equation (4) that the angles of the ray path relative to the magnetic field increase with frequency. As a spacecraft approaches the radiation source from left, the highest radiation frequency $f_{3}$ is then received first, followed by lower and lower frequencies, i.e., $f_{2}$ and $f_{1}$. The resulting frequency time variation is shown by the curved V-shaped lines in the bottom panel of Figure 6. If instead of a point source, the radiation is generated by a line source that extends upward along the magnetic field from the point marked "source," the result will be a frequency time spectrum that is filled in as shown by the shaded region. A field-aligned sheet source also leads to a filled-in spectrum, although the detailed intensity distribution within the filled-in region will be different than for a line source. Note that the existence of the low-frequency cutoff is related to the fact that the source has a sharply defined lower boundary.

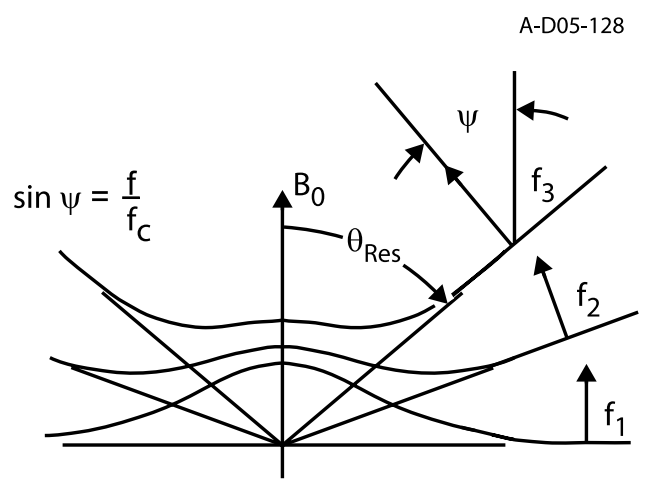

Figure 5. Polar plots of the surface of the index of refraction with frequencies $f_{1}, f_{2}$, and $f_{3}$. Here $\theta_{\text {Res }}$ is the resonance cone angle when the index of refraction $n(\theta)$ goes to infinity, and $\psi$ is the angle between limiting ray path direction and the magnetic field. 


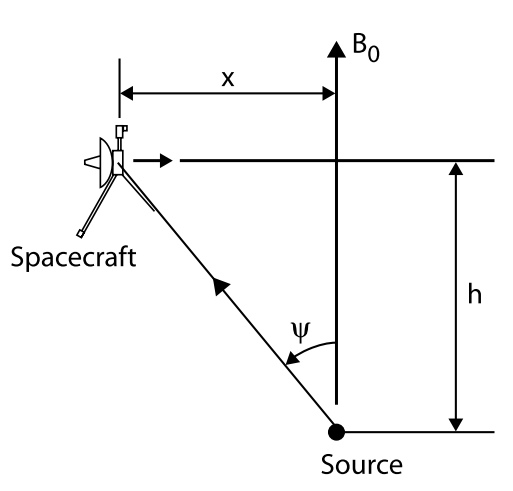

(a)

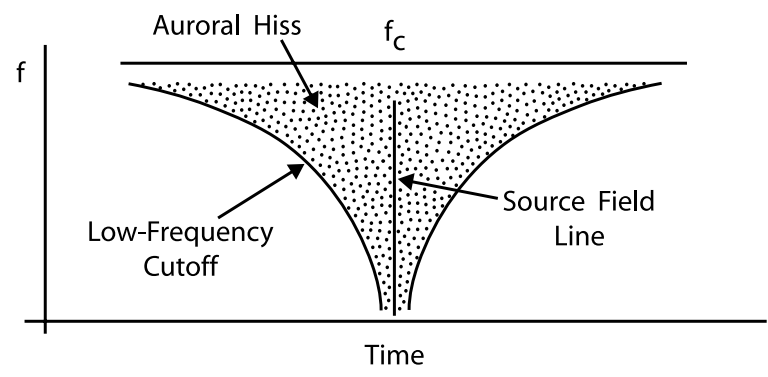

(b)

Figure 6. Demonstration of the geometry to produce a funnel-shaped low-frequency cutoff.

[11] To carry out a simplified analysis of the auroral hiss spectrum in Figure 1, we introduce a coordinate $x$, which is the perpendicular distance from the spacecraft to the magnetic field line through the source and at a distance $h$ below the spacecraft trajectory as shown in the top panel of Figure 6. By assuming that the rays are straight lines, it can be shown that

$$
\tan \psi=\frac{x}{h} .
$$

From equation (3) it is easy to show that

$$
\tan \psi=\frac{f}{\sqrt{f_{c}^{2}-f^{2}}} .
$$

Eliminating tan $\psi$ from the above two equations then gives an equation for the low-frequency cutoff in terms of spatial position

$$
f^{2}=f_{c}^{2}-\frac{h^{2}}{x^{2}+h^{2}} f_{c}^{2}
$$

For a constant spacecraft velocity it is easy to see that this equation gives a hyperbola frequency-time shape that is qualitatively similar to the low-frequency cutoff of the auroral hiss in Figure 1. However, because the spacecraft trajectory for the Io flyby is not exactly perpendicular to the magnetic field line, a more detailed geometric analysis is needed to provide a good fit to the observed low-frequency cutoff. We also note that auroral hiss emission in Figure 1 is not symmetrical, i.e., it does not have the right-hand branch of the funnel shown in Figure 6. Possible explanations for this asymmetry will be considered later.

\section{Detailed Geometric Analysis}

[12] In the following, we compute the location of the source of the auroral hiss by finding the best fit to the funnel-shaped low-frequency cutoff using an exact geometric model that takes into account the spacecraft trajectory. Initially, we assume the radiation source is a simple point source. Later we investigate the possibility that the source is a line or a sheet source aligned along the magnetic field.

\subsection{A Simple Point Source}

[13] Recall that the ray path angle for waves propagating near the resonance cone is given by the equation $\sin \psi=f l f_{c}$, where $\psi$ is the angle between the ray path direction and the magnetic field, $f$ is the cutoff frequency, and $f_{c}$ is the electron cyclotron frequency. To compare the theoretical model with the observations, we first calculate the time dependence of the cutoff frequency from the geometry of the source relative to the spacecraft trajectory. Figure 7 shows the geometry used in computing $\psi$, the angle between the limiting ray path and the magnetic field through the emission source. The point $R(x, y, z)$ is an arbitrary point along the trajectory, $R\left(x_{s}, y_{s}, z_{s}\right)$ represents the position of the source, and $R_{0}\left(x_{0}, y_{0}, z_{0}\right)$ is the point where the spacecraft passes through the magnetic line through the source. The magnetic field, $B_{0}$, is assumed to be constant and uniform and in the average direction of the magnetic field at 0119:30 UT, right in the center of the current sheet. Obviously, $R_{0}$ and $R_{s}$ are adjustable points. $R_{0}$ is the point where the lowest emission frequency occurs. By inspecting the spectrum in Figure 1, we find that the low-frequency apex of the emission occurs at about 01:20: 00 UT, which corresponds to the spacecraft coordinates $\left(x_{0}, y_{0}, z_{0}\right)=$ $(-0.893,0.24,-1.019)$. With this observation we fix the point $R_{0}$. Next the height of the source $h=\left|\boldsymbol{R}_{0}-\boldsymbol{R}_{s}\right|$ is adjusted until a best-fit to the low-frequency cutoff boundary is found. Figure 8 shows the results for three different values of $h$. The solid points in Figure 8 are from the measured low-frequency cutoff. From the plot in Figure 8,

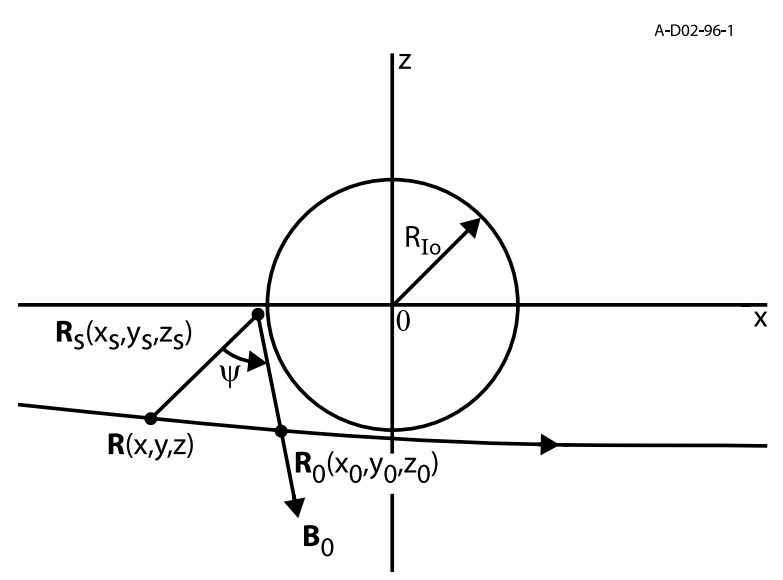

Figure 7. Demonstration of the geometry for locating the point source position when using best-fit propagation cutoff. 


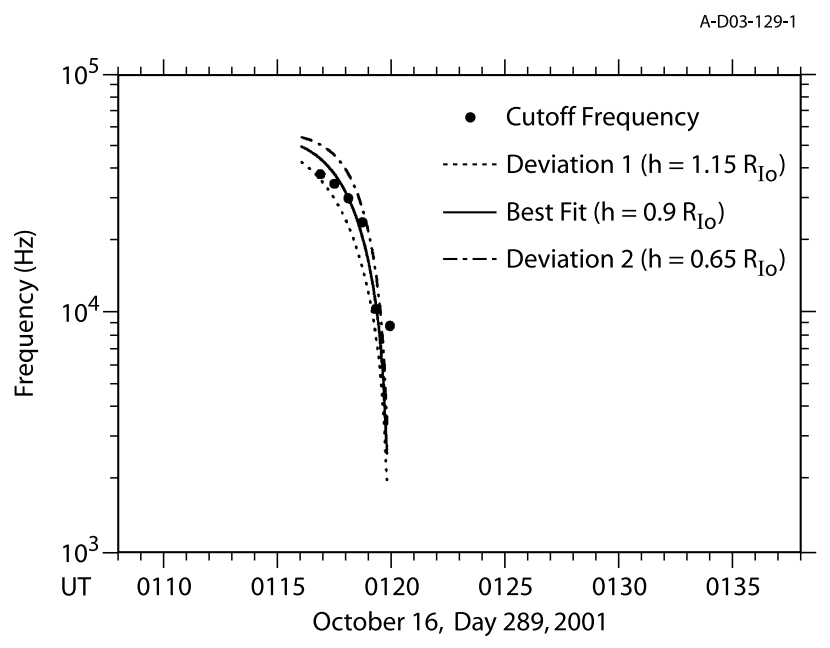

Figure 8. Fitting curves for three different point source positions. The solid circles are sampled from the cutoff emission boundary of the spectrum; three fitting curves are calculated for three different source positions.

we can see that the source is located roughly between $h=$ 0.65 and $h=1.15$, where $h$ is in the unit of the Io radii of Io. The wide range of $h$ indicates that the source does not have a sharply defined low-altitude boundary. This is reasonable since the emission spectrum in Figure 1 does not have a sharply defined low-frequency cutoff. The best-fit to the cutoff frequency gives $h=0.9$, which corresponds to a source at coordinates $R_{s}\left(x_{s}, y_{s}, z_{s}\right)=(-1.035,0.469,-0.16)$. This corresponds to an altitude of $0.15 \mathrm{R}_{\mathrm{Io}}$ above the surface of Io. The small value of $z_{s}=0.16 \mathrm{R}_{\mathrm{Io}}$ indicates that the source lies in the ionosphere very close to the equator of Io, roughly in the region where Jupiter's magnetic field is tangent to the surface of Io. Figure 9 shows the best-fit curve to the low-frequency cutoff for a source at $h=0.9 \mathrm{R}_{\mathrm{Io}}$ above the Galileo spacecraft. The good fit provides strong verification that the auroral hiss is propagating at wave normal angles near the resonance cone.

\subsection{A Cylindrical Sheet Source}

[14] Since the frequency-time spectrum of the radiation is filled in instead of being a sharp line, it is likely that the source of the emission is either a line or a sheet source. Applying the unipolar inductor model, we consider the possibility that the source is a cylindrical current sheet. We assume the axis of the cylindrical sheet is in the direction of the magnetic field direction at 0119:30 and that it passes through the center of the Io with radius

$$
r=\sqrt{x_{s}^{2}+y_{s}^{2}+z_{s}^{2}}=1.15 \mathrm{R}_{\mathrm{Io}} .
$$

The radius of the cylinder, $1.15 \mathrm{R}_{\mathrm{Io}}$ was chosen so that the cylinder passes through the source position that was determined from the simple point source model. Since we have no information of the magnetic field away from the spacecraft trajectory, we simply assume that the local current sources responsible for the radiation are also along the axis of the cylinder. Figure 10 shows the lower cutoff

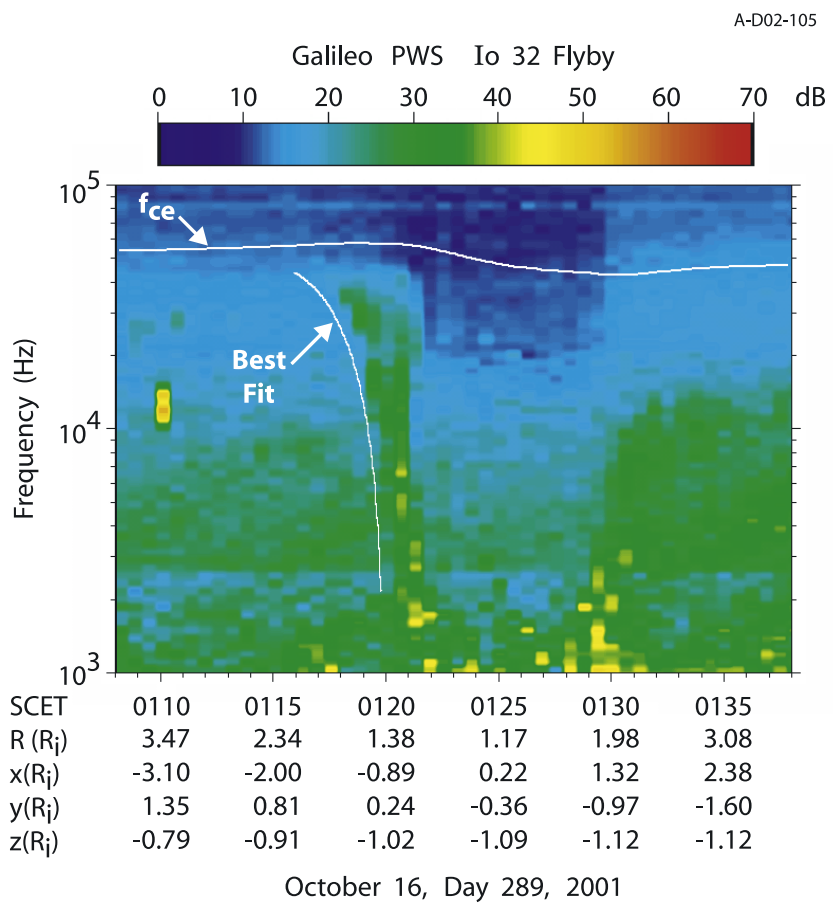

Figure 9. The best-fit curve for a source position with a distance $h=0.9 \mathrm{R}_{\text {Io }}$ above the Galileo spacecraft.

fitting curve calculated by assuming a cylindrical sheet source. The solid circles are the measured low-frequency cutoff frequencies, and the solid line is the fitting curve calculated as described above. As can be seen, the ray tracing fit to the observed spectrum is quite good, slightly better than for the point source model. Since the observed spectrum fills in the region within this outer boundary, it is highly likely that the source is an extended field-aligned

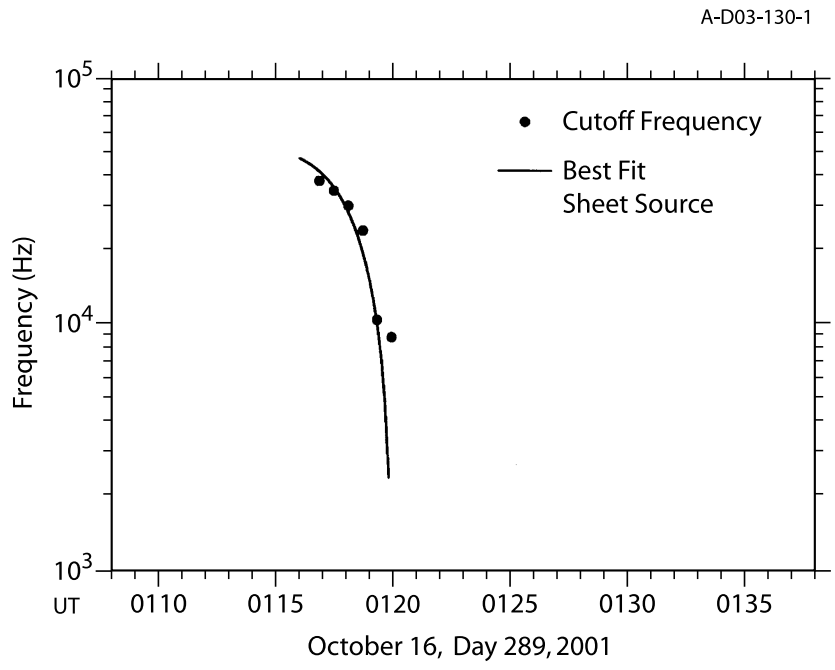

Figure 10. Cutoff frequency calculated from a sheet emission source (solid line) versus the sampled cutoff data (solid circles). 
sheet source. The good fit again provides a strong verification that the emission is propagating in the whistler mode at angles very close to the resonance cone, as has been assumed.

\section{Summary and Conclusion}

[15] Auroral hiss emissions have been observed by the Galileo spacecraft near Jupiter's moon Io. The frequency of the emission is below the local electron cyclotron frequency and electron plasma frequency and above the lower hybrid frequency. In this frequency range the only allowed plasma wave mode is the whistler mode, so the radiation must be propagating in the whistler mode. The electric field spectrum has a funnel-shaped low-frequency cutoff characteristic that is very similar to the terrestrial auroral hiss commonly observed in Earth's auroral region. The close association of the central axis of the funnel with the onset of the magnetic field perturbation caused by Io indicates that the emission is closely associated with a field-aligned current sheet originating from Io. This current sheet is most likely produced by the unipolar inductor interaction of Io with the Jovian magnetic field. Since auroral hiss is known to be produced by electron beams, these observations indicate the existence of a southward directed field-aligned electron beam accelerated in the ionosphere near Io. This means that the direction of the current is northward, pointing toward Io. This direction agrees well with that predicted by the unipolar inductor model of Goldreich and LyndenBell [1969] or the Alfvén wing model [Neubauer, 1980; Southwood et al., 1980]. The fact that no auroral hiss was observed during the outbound current sheet crossing is also consistent with these models, since any ionospheric electrons associated with this current sheet would be accelerated northward toward Io and would not produce observable radiation at the spacecraft.

[16] The main difference compared to terrestrial auroral hiss is that the spectrum is asymmetrical, i.e., the funnel does not have a right-hand branch. Such asymmetrical funnel-shaped auroral hiss emissions are occasionally observed in Earth's auroral zone. Currently, we have no definite explanation for this asymmetry. One possible explanation is that the plasma inside the flux tube is hot compared to the plasma outside the flux tube. High temperatures could cause the emission to be absorbed by Landau damping. We also note that there is an abrupt increase in the plasma density, hence electron plasma frequency coincident with the inbound current sheet crossing. It is possible that this gradient in the plasma density may play some role in preventing the radiation from entering the region of higher density over the south pole of Io.

[17] A series of ray-tracing computations has been performed by assuming a point and cylindrical sheet emission sources. It is found that the low-altitude boundary of the auroral hiss source lies near the equatorial plane at a height of about $270 \mathrm{~km}$ above the surface of Io. From the ionospheric electron density profile given by Kliore et al. [1974], we can see that the source lies well in the ionosphere of Io in a region where the local electron density is about $3 \times 10^{4}$ electrons per cubic centimeter. The generation mechanism of this type of emission is commonly believed to be a beam-driven plasma instability associated with the Landau resonance at $v_{\|}=\omega / k_{\|}$. The existence of a sharp low-altitude boundary to the source strongly suggests that the electron beam may be accelerated at this point, possibly by parallel electric fields similar to those that are known to form in terrestrial field-aligned currents that link the auroral regions to the distant magnetosphere.

[18] Acknowledgments. Research at the University of Iowa was supported by NASA through grant NNG05GG98G.

[19] Arthur Richmond thanks the reviewers for their assistance in evaluating this paper.

\section{References}

Bagenal, F. (1994), Empirical model of the Io plasma torus: Voyager measurements, J. Geophys. Res., 99, 11,043-11,062.

Cartwright, D. G., and P. J. Kellogg (1974), Observation of radiation from an electron beam artificially injected into the ionosphere, J. Geophys. Res., 79, 1439-1457.

Ellis, G. R. A. (1957), Low-frequency radio emission from aurorae, J. Atmos. Terr. Phys., 10, 302-306.

Farrell, W. M., D. A. Gurnett, P. M. Banks, R. I. Bush, and W. J. Raitt (1988), An analysis of whistler-mode radiation from the Spacelab 2 electron beam, J. Geophys. Res., 93, 153-161.

Farrell, W. M., D. A. Gurnett, and C. K. Goertz (1989), Coherent Cerenkov radiation from the Spacelab 2 electron beam, J. Geophys. Res., 94, 443452.

Farrell, W. M., R. J. MacDowall, R. A. Hess, M. L. Kaiser, M. D. Desch, and R. G. Stone (1993), An interpretation of the broadband VLF waves near the Io torus as observed by Ulysses, J. Geophys. Res., 98, 21,17721,188 .

Goldreich, P., and D. Lynden-Bell (1969), Io, a Jovian unipolar inductor, Astrophys. J., 156, 59-78.

Gurnett, D. A. (1964), High-latitude geophysical studies with satellite Injun 3, J. Geophys. Res., 69, 65-89.

Gurnett, D. A. (1966), A satellite study of VLF hiss, J. Geophys. Res., 71, $5599-5615$

Gurnett, D. A., and A. Bhattacharjee (2005), Introduction to Plasma Physics with Space and Laboratory Applications, p. 119, Cambridge Univ. Press, New York.

Gurnett, D. A., and L. A. Frank (1972), VLF hiss and related plasma observations in the polar magnetosphere, J. Geophys. Res., 77, 172-190.

Gurnett, D. A., and W. S. Kurth (1979), Auroral hiss observed near the Io plasma torus, Nature, 280, 767-770.

Gurnett, D. A., S. D. Shawhan, and R. R. Shaw (1983), Auroral hiss, Z-mode radiation, and auroral kilometric radiation in the polar magnetosphere: DE 1 observations, J. Geophys. Res., 88, 329-340.

Gurnett, D. A., W. S. Kurth, J. T. Steinberg, P. M. Banks, R. I. Bush, and W. J. Raitt (1986), Whistler-mode radiation from the Spacelab 2 electron beam, Geophys. Res. Lett., 13, 225-228.

Helliwell, R. A. (1965), Whistlers, p. 30, Stanford Univ. Press, Stanford, Calif.

Jackson, J. D. (1962), Classical Electrodynamics, John Wiley, Hoboken, N. J.

James, H. G. (1973), Whistler mode hiss at low and medium frequencies in the dayside cusp, J. Geophys. Res., 78, 4578-4599.

Jorgensen, T. S. (1968), Interpretation of auroral hiss measured on OGO-2 and at Byrd Station in terms of incoherent Cerenkov radiation, J. Geophys. Res., 73, 1055-1069.

Kivelson, M. G., K. K. Khurana, R. J. Walker, J. Warnecke, C. T. Russell, J. A. Linker, D. J. Southwood, and C. Polanskey (1996), Io's interaction with the plasma torus: Galileo magnetometer report, Science, 274, 396-398.

Kliore, A., D. L. Cain, G. Fjeldbo, B. L. Seidel, and S. I. Rasool (1974), Preliminary results on the atmospheres of Io and Jupiter from Pioneer 10 S-band occultation experiment, Science, 183, 323-324.

Maggs, J. E. (1976), Coherent generation of VLF hiss, J. Geophys. Res., 81, $1707-1724$

Martin, L. H., R. A. Helliwell, and K. R. Marks (1960), Association between aurorae and very-low-frequency hiss observed at Byrd Station, Antarctica, Nature, 187, 751-753.

Monson, S. J., P. J. Kellogg, and D. G. Cartwright (1976), Whistler-mode plasma waves observed on Electron Echo 2, J. Geophys. Res., 81, $2193-$ 2199

Morgan, D. D., D. A. Gurnett, W. S. Kurth, and F. Bagenal (1994), The source of Jovian auroral hiss observed by Voyager 1, J. Geophys. Res., 99, 21,213-21,224.

Mosier, S. R., and D. A. Gurnett (1969), VLF measurements of the Poynting flux along the magnetic field with the Injun 5 satellite, J. Geophys. Res., $74,5675-5687$. 
Ness, N. F., M. H. Acuna, R. P. Lepping, L. F. Burlaga, K. W. Behannon, and F. M. Neubauer (1979), Magnetic field studies at Jupiter by Voyager 1: Preliminary results, Science, 203, 42-46.

Neubauer, F. M. (1980), Nonlinear standing Alfvén wave current system at Io: Theory, J. Geophys. Res., 85, 1171-1178.

Southwood, D. J., M. G. Kivelson, R. J. Walker, and J. A. Slavin (1980), Io and its plasma environment, J. Geophys. Res., 85, 5959-5968.

Stix, T. H. (1992), The Theory of Plasma Waves, McGraw-Hill, New York.
Taylor, W. W. L., and S. D. Shawhan (1974), A test of incoherent Cerenkov radiation for VLF hiss and other magnetospheric emissions, J. Geophys. Res., 79, 105-117.

D. A. Gurnett and L. Xin, Department of Physics and Astronomy, University of Iowa, Iowa City, IA 52242, USA. (lei-xin@uiowa.edu)

M. G. Kivelson, Institute of Geophysics and Planetary Physics, University of California, Los Angeles, Los Angeles, CA 90095, USA. 